\title{
A Counter Prechop Technique Using a Modified Universal Prechopper in Combination with or without Using a Universal Chopper
}

\author{
Hyungil Kim', Younghoon Jung $\mathbb{D}^{2}$, Mikio Inamura ${ }^{3}$ \\ 'Gyeongju St. Mary's Eye Clinic, Gyeongju, Republic of Korea; ${ }^{2}$ Department of Ophthalmology, Pohang Naval Hospital, Pohang, Republic of Korea; \\ ${ }^{3}$ Inamura Eye Clinic, Yokohama, Japan \\ Correspondence: Mikio Inamura, Inamura Eye Clinic, 5-I25 Isesakicho, Naka-ku, Yokohama, Kanagawa, 23I-0045, Japan, Email m-ina@sa2.so-net.ne.jp
}

Purpose: To introduce an alternative prechop technique without or with the use of a modified universal chopper (AE-2591, HI Kim chopper, ASICO, Inc. USA).

Methods: Patients who underwent cataract surgery using the phaco-chop technique and the modified prechop technique have been grouped and reviewed retrospectively. Endothelial cell count (ECC) and central corneal thickness (CCT) measured 7 days preoperatively, and 1 month and 3 months post-operatively, were compared between the two groups. In modified prechop technique, a narrow $1.7 \mathrm{~mm}$ neck prechopper (AE-4298, HI Kim-Inamura chopper, ASICO, Inc., USA) was used with universal chopper (AE2591, HI Kim chopper, ASICO, Inc., USA) as an additional device for grade 4 and above cataracts or without it for grade 2 to 3 cataracts. Details of the surgical technique is further described in the Method section.

Results: A total of 104 eyes in the modified prechop group and 97 eyes in the phaco-chop group were enrolled in the study. Preoperative nuclear opacity, ECC, and CCT as well as post-operative ECC (absolute value and the loss) and CCT (absolute value and the change) throughout the follow-up between the two groups did not show statistically significant differences.

Conclusion: Counter prechop technique using the modified prechopper and universal chopper is a safe, easy, and versatile surgical technique that can be applied broadly in a wide range of cases with various complexities, including LOCS 111 N1 to N6 nuclei, hypermature cataracts, small pupils, and fragile suspensory zonular ligaments, with post-operative result not inferior to that of the conventional technique.

Keywords: prechop, counter prechop, cataract

\section{Introduction}

Phacoemulsification is one of the most widely used cataract surgery techniques that was initially introduced by Kelman ${ }^{1}$ in 1967, which stabilized anterior chamber intraoperatively, shortened post-operative astigmatism, and expedited recovery by making an incision smaller than the conventional extracapsular cataract extraction (ECCE). However, phacoemulsification requires high-power ultrasound (US) energy, thus increasing the risk of anterior and posterior capsular ruptures, corneal endothelial cell loss, zonular stress, prolonged surgical time, and complications such as decreased visual acuity.

The horizontal chop technique introduced by Nagahara is another method that has been considered safe and effective where opposing forces created by the chopper split the lens nucleus. This technique also requires a significant level of ultrasound energy during nucleus disassembly, although it is less than that of the divide-and-conquer technique. The horizontal chop technique can induce fluid turbulence in balanced salt solution (BSS), which increases the risk of anterior chamber instability, endothelial cell damage, and miosis. Alternatively, the vertical phaco-chop (phaco quick chop, or karate phaco-chop) technique has been adopted in cases with hard cataracts, small pupils, and small anterior capsular incisions to minimize the US energy exposure. 
The abovementioned surgical techniques all require a lengthy training period to master each and one of them, during which surgeons often encounter numerous surgical challenges. The intricate process of skill development occasionally acts as a barrier to surgeons learning a new technique once they become familiarized with a certain approach adapted and individualized to confidently perform the surgery. However, operating on nucleus disassembly and phacoemulsification concurrently can raise various risks in complex cases, such as moderate to severe cataract, shallow anterior chamber depth, small pupils, unstable incision, or weak ciliary zonule. Stemming from these concerns was the call for a new technique that was not only easy to learn, but also stable with separate nucleus disassembly and phacoemulsification processes, and at the same time requiring minimal US energy and BSS. The manual prechop technique, first introduced by Akahoshi $^{2}$ in 1998, removed the use of US energy in fragmentation and significantly reduced both US energy exposure and surgical time when compared with conventional chopping procedures such as divide-and-conquer, ${ }^{3}$ stopand-chop, ${ }^{4}$ or the supracapsular flip ${ }^{5}$ technique.

The Akahoshi prechopper, a type of combo prechopper, can be used in LOCS III N1 to LOCS III N3 cataracts without raising the risk of zonulopathy. For LOCS III N4 and above lenses, other types of prechopper as well as an Inamura prechopper can be used. However, such an approach not only increases zonular stress, but also requires additional instruments, such as a blunt nucleus manipulator or a sustainer to create counterforce, which adds extra risk of zonular stress when incomplete or mis-directed separation occurs. Finally, conventional prechop techniques increase the risk of posterior capsular damage or zonular weakness in difficult cases, such as LOCS III N5 and above nucleus, mature or hypermature cataracts with hard nucleus, or preexisting zonular weakness.

The modified narrow $1.7 \mathrm{~mm}$ neck prechopper (AE-4298, HI Kim-Inamura prechopper, ASICO, Inc., USA) aims to overcome limitations of conventional techniques. It allows lens division in both soft and dense nucleus without exerting a counterforce. The narrow tip is suitable for microincision cataract surgery (MICS) with $1.8 \mathrm{~mm}$ or $2.0 \mathrm{~mm}$ incision. The wide tip blade is designed to split the nucleus with uniformly distributed force, reducing the risk of ciliary zonular damage from shearing force when the blade is widened after inserted to the lens nucleus. In addition, the eagle's beak shaped tip allows the surgeon to maintain a steady hold of the nucleus fragment while providing a steady force to split the lens. For mature cataracts with hard nucleus, the universal chopper (AE-2591, HI Kim chopper, ASICO, Inc., USA) can be used instead of additional equipment, such as blunt nucleus manipulator or a sustainer, to perform counter prechop as well as conventional horizontal chop or stop-and-chop techniques.

This article aims to introduce an alternative prechop technique using HI Kim-Inamura prechopper and universal chopper which can be applied safely and effectively in LOCS III N4 or higher-grade cataracts even with a thin cortical layer. The technique not only stabilizes fixation by applying evenly increased chopping force, but also separates the use of phacoemulsification instruments in lens fragmentation process, intending to minimize intraoperative and postoperative complications.

\section{Materials and Methods}

\section{Patients}

Patients with age-related cataracts who underwent cataract surgeries using modified prechop technique or phaco-chop phacoemulsification nucleotomy techniques were enrolled in this study from Gyeongju St. Mary's eye clinic, Gyeongjusi, Gyeongsangbuk-do, Republic of Korea, from May 1, 2016 to April 30, 2017 (for Phaco-chop group) and from September 1, 2020 to August 30, 2021 (for Modified prechop group). The grading of the cataract was evaluated based on the Lens Opacities Classification System III (LOCS III) by the same physician. Eyes with nuclear opacity graded between $2+$ and $6+$ were included for analysis in this study. The exclusion criteria included a history of ocular trauma or ophthalmic surgery, ocular comorbidities, corneal disorders, and an endothelial cell count (ECC) of less than 1500 cells/ $\mathrm{mm}^{2}$. Patients without regular follow-up were excluded as well. This study was approved by the Institutional Review Board of Gyeongju St. Mary's eye clinic (GMS-202112) and followed the principles of the Declaration of Helsinki. Informed consent was obtained from each patient. 


\section{Patient Evaluation}

All patients received a full ophthalmic examination, which included the best-corrected distance visual acuity, manifest refraction, slit-lamp examination, intraocular pressure measurement (NT-800, Topcon, Tokyo), ECC, central corneal thickness (CCT) by a non-contact specular microscope (SP-1P, Topcon, Tokyo), and biometry by A-mode ultrasound (Aviso, Quantel Medical) or coherence interferometry (IOLMaster, Carl Zeiss Meditec AG) and fundus examination. All patients underwent the same pre-operative standardized management.

\section{Phaco Machine and Settings}

The Stellaris $\mathrm{PC}^{\circledR}$ Phacoemulsification unit (Bausch\&Lomb, St. Louis, MO, USA) and the outer diameter $0.74 \mathrm{~mm}-0.94 \mathrm{~mm}$ flared, $30^{\circ}$ angulated needle, straight $1.8 \mathrm{~mm}$ MICS ${ }^{\mathrm{TM}}$ needle were used for each cataract surgery in the modified prechop group. The settings of the phaco machine were as follows: the longitudinal multiple burst mode (burst duration: 6/duty cycle: 75 ) with $30 \%$ ultrasound power, infusion bottle height of $80 \mathrm{~cm}$, and vacuum 430 $\mathrm{mmHg}$.

The Infiniti ${ }^{\circledR}$ phacoemulsification unit (Alcon Laboratories, Fort Worth, TX, USA) and the $0.9 \mathrm{~mm}$ Reverse Kelman microtip were used for each cataract surgery in the phaco-chop control group. The settings of the phaco machine were as follows: the torsional continual model with $100 \%$ amplitude, infusion bottle height of $100 \mathrm{~cm}$, aspiration flow rate of $38 \mathrm{~mL} / \mathrm{min}$, and vacuum $430 \mathrm{mmHg}$.

\section{Surgical Technique}

All the surgeries were performed under topical anesthesia by one experienced surgeon (Hyungil Kim), who had previously used the modified prechop technique in more than 5000 cases and performed more than 25,000 cataract surgeries prior to this study.

Under topical anesthesia, a $1.8-2.0 \mathrm{~mm}$ or a standard $2.2 \mathrm{~mm}$ transparent corneal incision is made along the steepest axis. A viscoelastic agent is injected into the anterior chamber. A continuous curvilinear capsulorhexis is performed using the capsulorhexis forceps. The average capsule opening diameter is $5.5-5.8 \mathrm{~mm}$ and depends on different types of IOLs. As capsulorhexis has been completed, the cortical cleaving hydrodissection is performed using a 23-gauge dual port cannula (AE-7655, HI Kim-Inamura cannula, ASICO, Inc., USA), which injects BSS in different directions with minimal zonule stress and allows the nucleus to rotate freely in the capsular bag (Figure 1) (Video 1).

In the case of cataracts with relatively soft nucleus (below grade 3), nucleus fragmentation is conducted in the same way as the conventional prechop maneuver. After filling the anterior chamber with enough dispersive ophthalmic viscosurgical devices (OVD), insert the modified narrow $1.7 \mathrm{~mm}$ neck prechopper (AE-4298, HI Kim-Inamura prechopper, ASICO, Inc. USA) vertically and place it slightly past the anatomical Y suture at the center of the lens (Figure 2). When passing through the endonucleus, open the prechopper blades slowly and the nucleus can be easily divided. After rotating the nucleus into 90 degrees, chop the nucleus distally from the incision, followed by chopping the nucleus proximally to the incision and phacoemulsify the divided quadrants (Figure 3A-G) (Video 2). If the nucleus does not turn freely, nuclear chopping can be performed radially around the incision (Figure 4) (Video 3).

In the case of cataracts with hard nucleus (above grade 4), nucleus fragmentation is performed using the counter prechop technique, introduced as in following steps. Insert the $2.0 \mathrm{~mm}$ universal chopper with a rounded tip (AE 2591, HI Kim chopper, ASICO, Inc.) into the anterior chamber through the $1.0 \mathrm{~mm}$ paracentesis incision, slide it through the cortical layers, and position it at the nucleus equator, while the prechopper is placed at 180 degrees opposite just before the anatomical Y suture. As the universal chopper is pulled centrally with slightly upward force, simultaneously push the opposite prechopper centrally with slight downward force. Both instruments should be handled with the same amount of force and should approach each other slowly (Figure 5A-E) (Videos 4 and 5). The prechopper should be placed at the center of the nucleus, and gently opening the blades will fracture the hard endonucleus. If additional nuclear fracturing is required, the prechopper can be flipped over and placed under the center of the lens. By opening the blades, the nuclear fragmentation is successfully completed (Figure 6). The nucleus is divided into 4 or more fragments using universal chopper with prechopper and followed by phacoemulsification. 


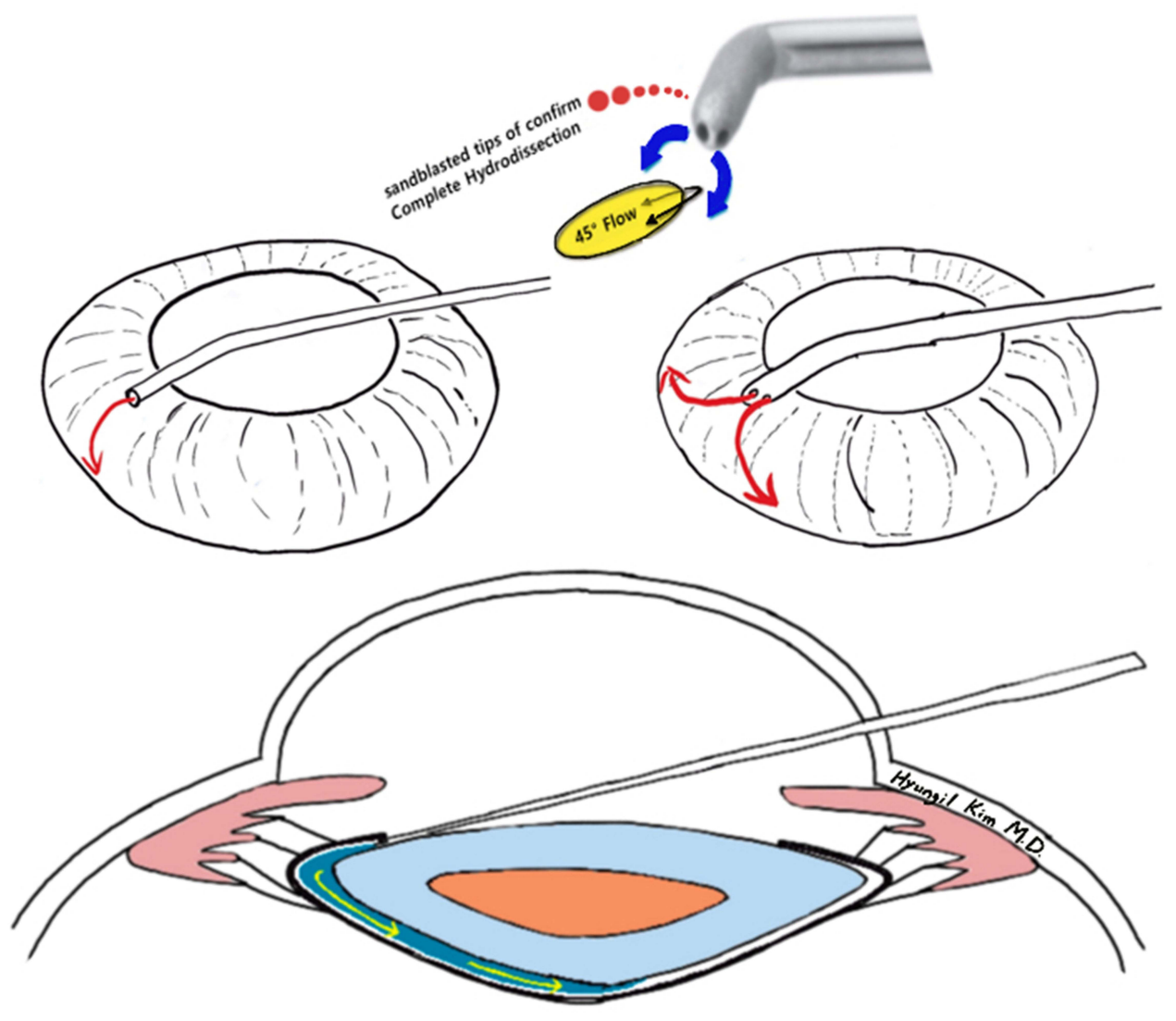

Figure I The illustration of 23-gauge dual port cannula, which injects BSS in different directions.

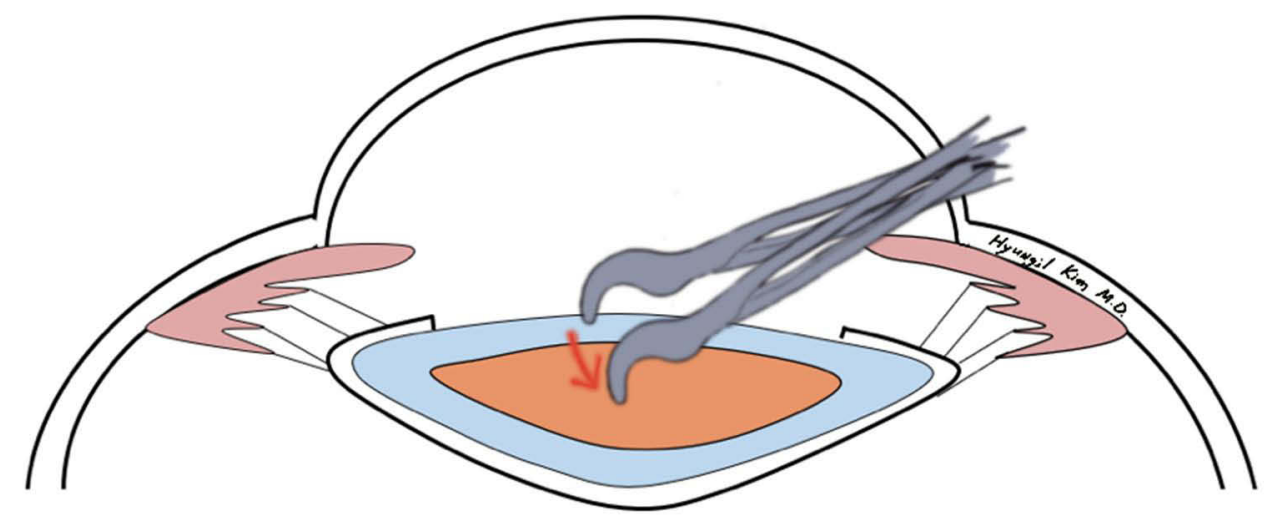

Figure 2 Cross-sectional schematic of the prechopping. Insert prechopper vertically and place it slightly past the anatomical $Y$ suture.

\section{Outcome Assessment}

The ECC was measured 7 days prior to surgery, as well as 1 month and 3 months after surgery. The CCT was measured 7 days before surgery, as well as 1 month and 3 month post-operatively. All the ophthalmic examinations were performed by the same technician. The percentage of endothelial cell loss was calculated as follows: Loss of ECC $(\%)=($ ECCpreECCpost)/ECCpre. The change in CCT was calculated as follows: Change in CCT $(\%)=(C C T p o s t-$ CCTpre)/CCTpre. Pre is short for pre-operative and post is short for post-operative. 


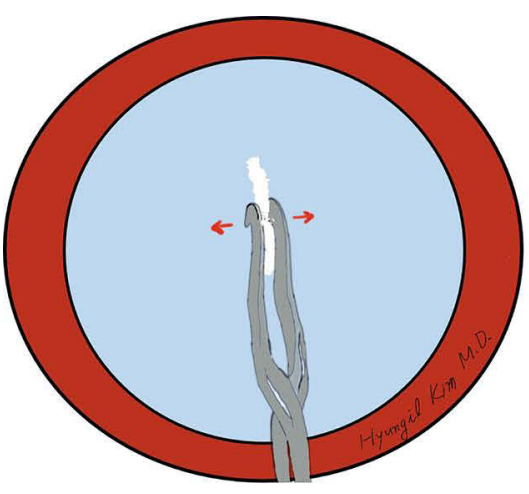

A

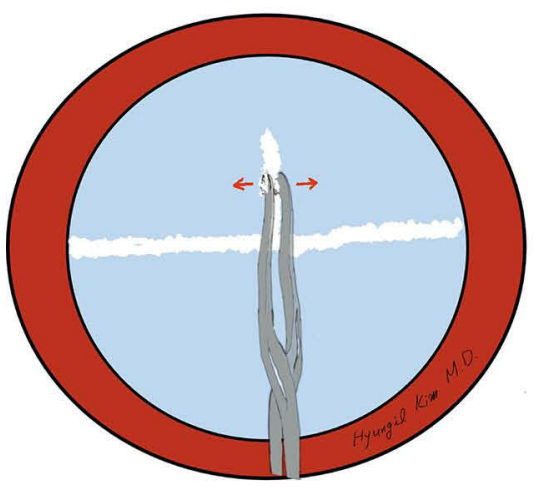

D

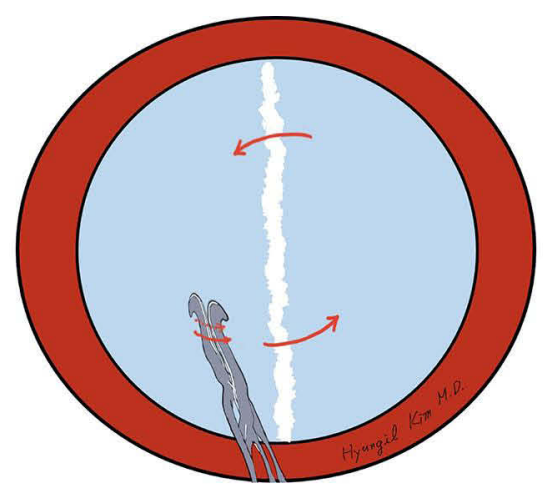

B

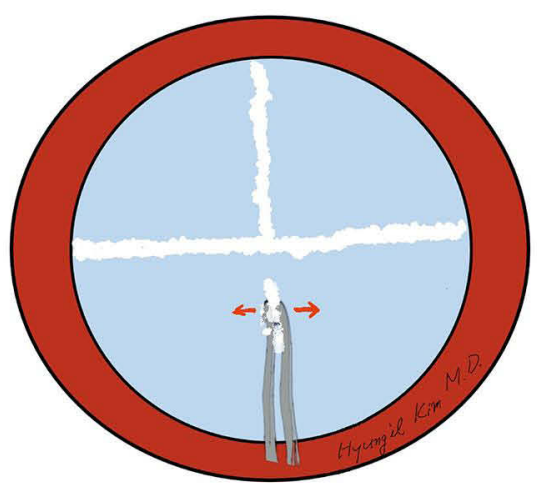

E

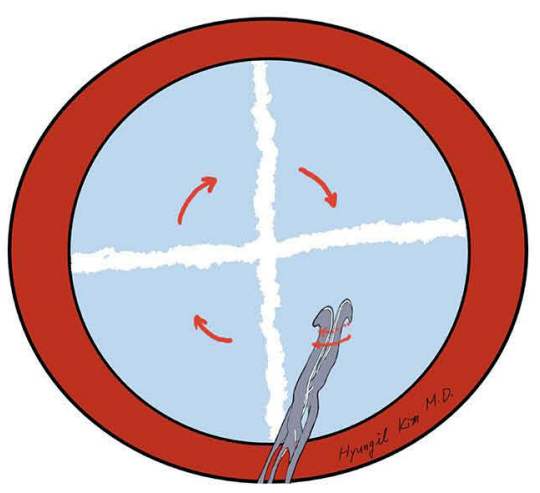

G

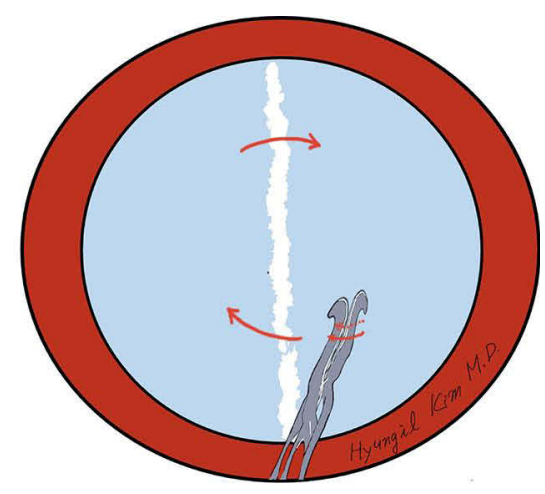

C

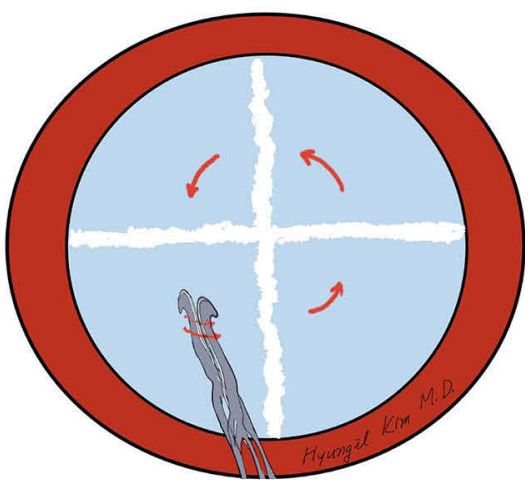

$\mathbf{F}$

Figure 3 (A) At the point where more than 2/3 of the inner nucleus of the lens has passed, slowly spread the prechopper blade to the side to split the nucleus. (B and C) The curved tip of the prechopper spans the nuclear surface vertically and rotates the lens 90 degrees clockwise or counterclockwise. (D) Secondary nuclear cleavage is additionally performed from the divided nucleus far away from the incision. (E) The nucleus close to the incision is also split and finalized into 4 equal parts. (F and $\mathbf{G})$ Turn the entire nucleus divided by 45 degrees to position it so that docking of the nucleus is easy during phacoemulsification.

\section{Statistical Analysis}

Statistical analysis was performed with SPSS software package version 18 (SPSS Inc., Chicago, Illinois, USA). Normally distributed variables were expressed as mean \pm standard deviation. The independent samples $t$-test was used to compare the mean values between the two groups, whereas paired $t$-test was used to compare the pre-operative and post-operative data. A chi-square test was applied to analyze the qualitative data. The threshold for statistical significance was set at $P<0.05$. 


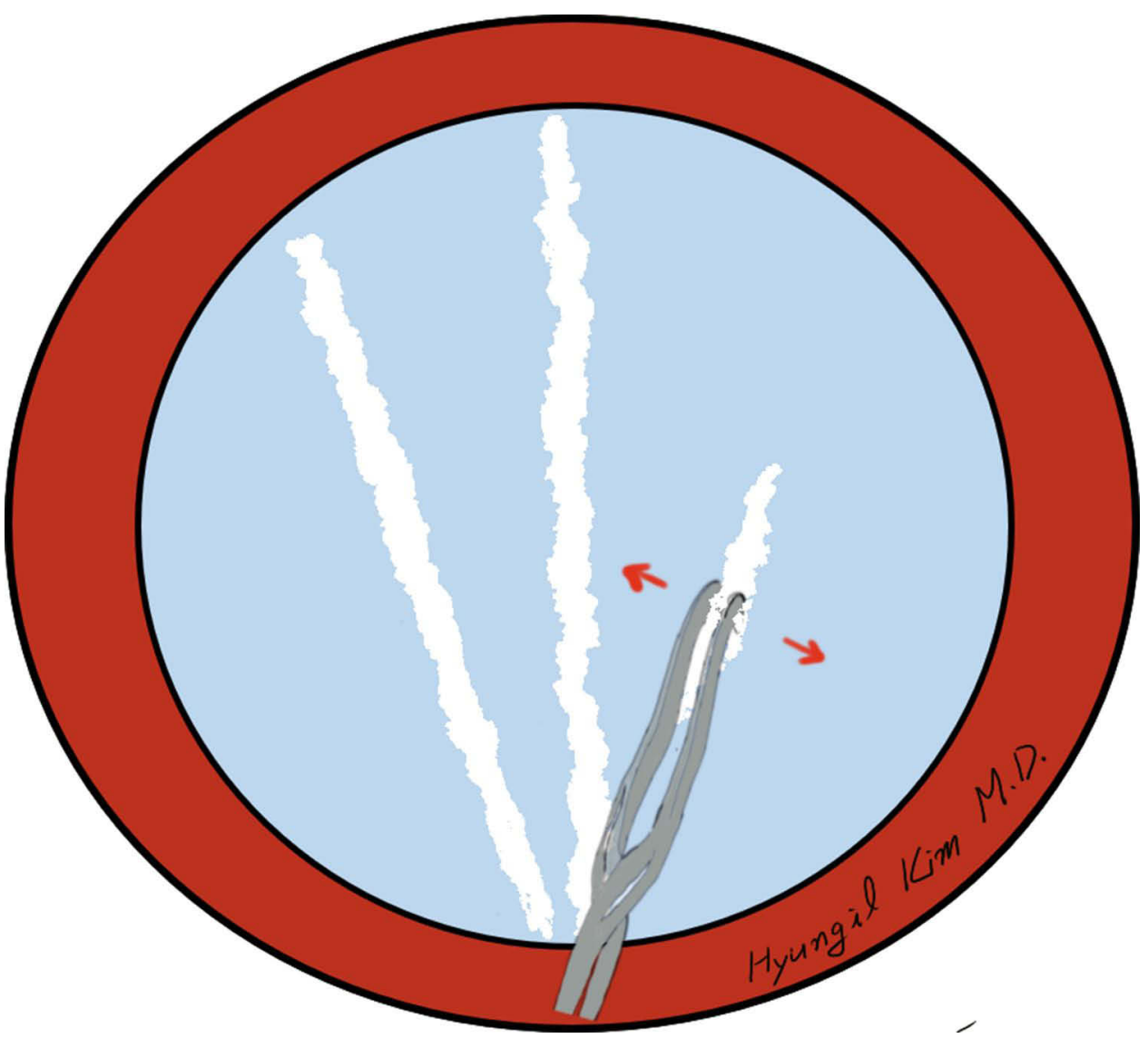

Figure 4 Schematic of the radial nuclear chopping using prechopper.

\section{Results}

A total of 201 eyes of 201 patients (104 eyes in the modified prechop group, and 97 eyes in the phaco-chop group) were enrolled for analysis in this study. The modified prechop group consisted of 104 eyes of 76 women and 28 men. The phaco-chop group consisted of 97 eyes of 57 women and 40 men. The demographic and clinical characteristics of the study (modified prechop) and control (phaco-chop) groups are presented in Table 1. The mean age of the modified prechop group was 70.1 \pm 10.9 years. There were significant differences between the two groups in gender and age; however, no significant difference in nuclear opacity was observed between the 2 groups. Also, no significant differences in the mean ECC (Table 2) and CCT (Table 3) pre-operatively were found between the two groups.

No statistically significant differences in the absolute ECCs and losses of ECC throughout the follow-up ( $P=0.307$, 0.375 , respectively, at 1 month; and $P=0.252,0.327$, respectively, at 3 months, Table 2 ) were observed between the two groups.

Likewise, no statistically significant differences in the absolute CCT and change in CCT throughout the follow-up ( $P=0.662,0.404$, respectively, at 1 month; and $P=0.618,0.272$, respectively at 3 months, Table 3 ) were observed between the two groups.

\section{Discussion}

Some studies have proposed different types of prechopping technique, using 1 or 2 cystotome, ${ }^{6,7}$ reverse type chopper, ${ }^{8}$ and phacoemulsification handpiece as a second chopper. ${ }^{9}$ Both middle prechop ${ }^{6}$ and 1 cystotome-assisted prechop ${ }^{7}$ have a possibility to potential iatrogenic injury during the introduction of the cystotomes through the incision. The main chopping force during middle prechop ${ }^{6}$ is towards the posterior capsule and zonule, which can lead to increased posterior capsular rupture and zonular dialysis or zonulopathy. The reverse type chopper ${ }^{8}$ is relatively hard to manipulate, especially for physicians with less clinical experience, and poor manipulation of this equipment can result in breakage 


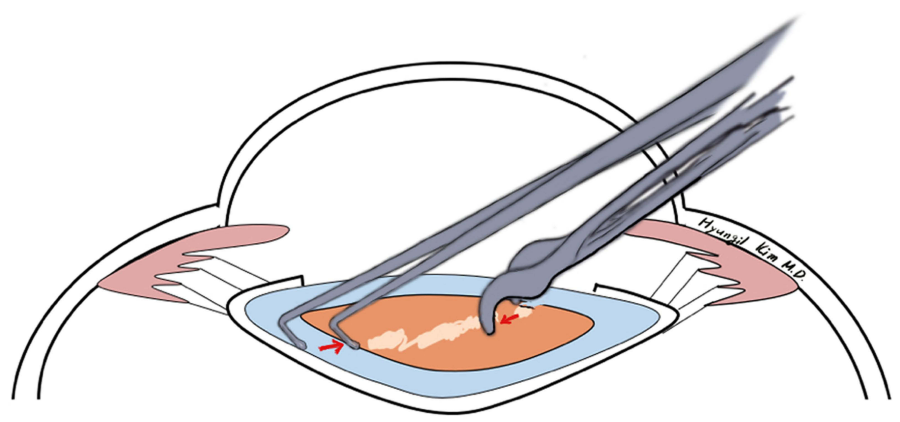

A

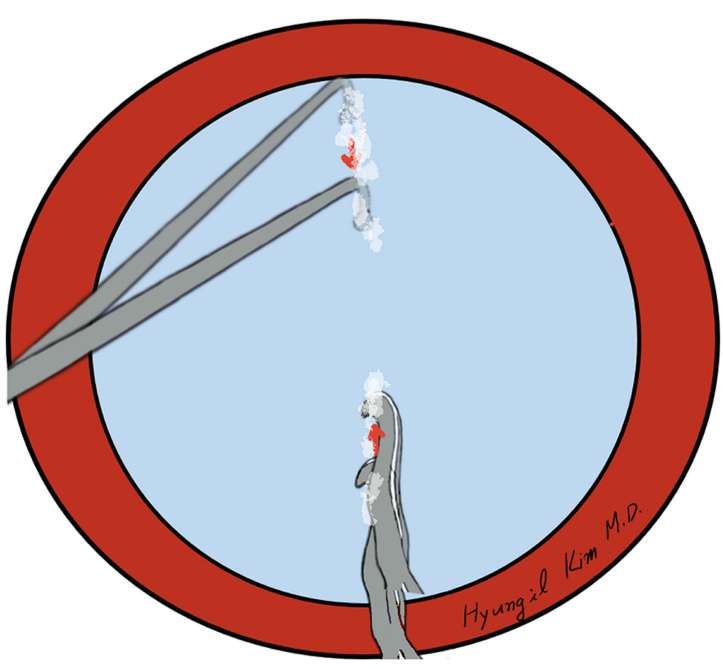

B

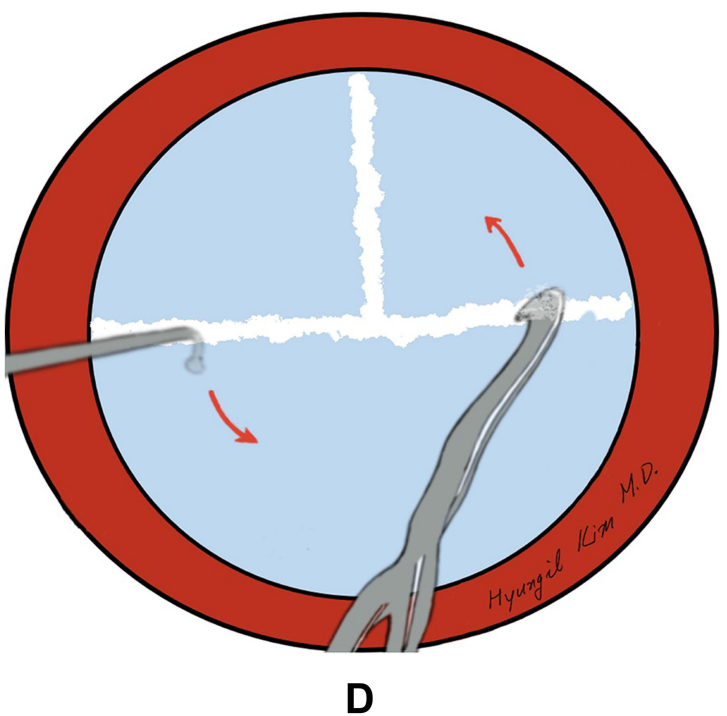

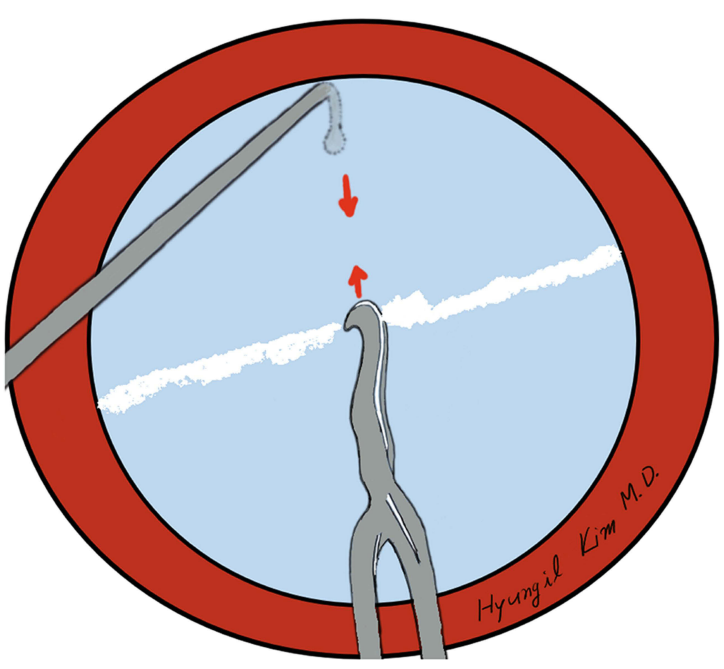

C

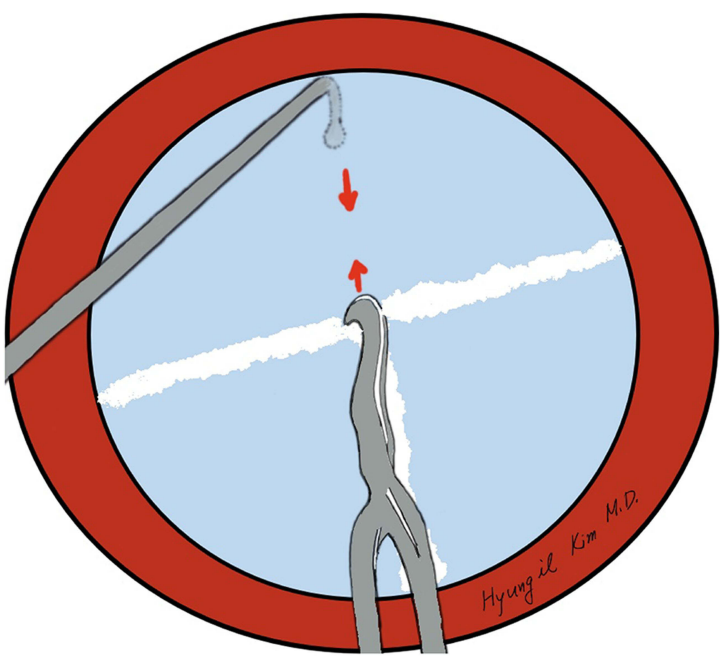

$\mathbf{E}$

Figure 5 (A and B) Cross-sectional schematic of the counter prechopping. Pull the chopper centrally with slightly upward force, simultaneously push the opposite prechopper centrally with slight downward force. (C) The same procedures like nuclear Ist nuclear chopping methods. (D) The rotation of the split nuclei with two instruments. (E) The final nuclear fracturing with the prechopper and the universal chopper. 


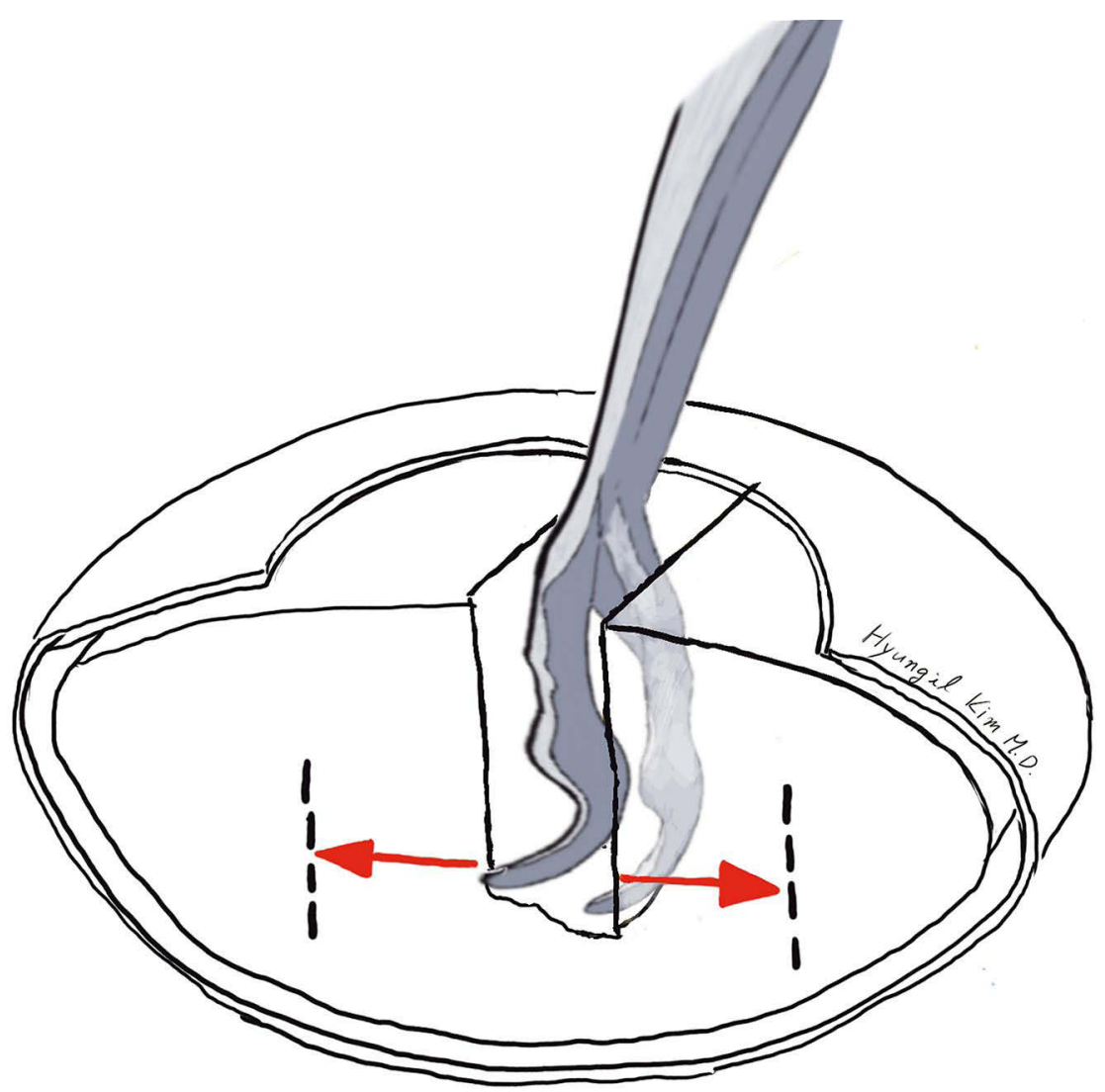

Figure 6 Schematic of the lens division using prechopper which can be flipped over.

of the posterior capsule. The double-chop maneuver suggested by $\mathrm{Kim}^{9}$ can fracture a wide range of lens densities, from very soft to dense and even in cases with small pupils; however, it does not entirely separate the use of phacoemulsification instrument and lens fragmentation.

When using double-chop maneuver, a clinician is required to continuously manipulate irrigation with a foot pedal from 0 to 1 , while simultaneously attempting to insert the chopper and phacoemulsification tips through the incision.

Also, after phaco tip and chopper are brought together at the center of the nucleus, lateral separation is necessary to divide the lens. Incomplete or mis-directed lateral separation can lead to increased zonular stress. Therefore, it can be challenging for physicians with less experience to carry out two hand counter prechop in every single case of cataract.

Since Nagy et al, ${ }^{10}$ some studies showed potential advantages of using femtosecond laser-assisted cataract surgery (FLACS), which includes reduction of endothelial cell loss, better IOL position, improved circularity of capsulotomies, less corneal swelling, and decreased effective phacoemulsification time. ${ }^{11-15}$ However, there are opposing study results that showed FLACS requiring prolonged operating time, ${ }^{16}$ and highlighting the additional costs of the procedure. ${ }^{17}$ Recent mid-term meta-analysis study showed that there were no significant differences found in visual acuity at 1 week

Table I Patient Demographics and Clinical Characteristics

\begin{tabular}{|l|c|c|c|c|}
\hline & Prechop Group & Phaco-chop Group & Total & $P$ \\
\hline Eyes & 104 & 97 & 201 & 0.032 \\
\hline Gender & $28 / 76$ & $40 / 57$ & $68 / 133$ & 0.001 \\
\hline Age & $70.1 \pm 10.9(44-89)$ & $75.2 \pm 9.6(53-91)$ & $72.6 \pm 10.5(44-91)$ & 0.546 \\
\hline LOCS III grading $(2 / 3 / 4 / 5 / 6)$ & $24 / 24 / 30 / 12 / 14$ & $16 / 26 / 23 / 16 / 16$ & & \\
\hline
\end{tabular}


Table 2 Comparison of Corneal Endothelial Cells Pre-Operatively and Post-Operatively in Modified Prechop and Phaco-Chop Groups

\begin{tabular}{|l|c|c|c|}
\hline & Prechop Group & Phaco-chop Group & $P$ \\
\hline Pre-operative (cells $/ \mathrm{mm}^{2}$ ) & $2679 \pm 263$ & $2653 \pm 241$ & 0.478 \\
\hline I-month post-operative & & & 0.307 \\
ECC (cells $/ \mathrm{mm}^{2}$ ) & $2354 \pm 423$ & $2288 \pm 491$ & 0.375 \\
Loss of ECC (\%) & $11.9 \pm 14.5 \%$ & $13.9 \pm 16.2 \%$ & \\
\hline 3-month post-operative & & & 0.252 \\
ECC (cells $\left./ \mathrm{mm}^{2}\right)$ & $2364 \pm 370$ & $2296 \pm 462$ & 0.327 \\
Loss of ECC (\%) & $11.4 \pm 12.7 \%$ & $13.4 \pm 15.7 \%$ & \\
\hline
\end{tabular}

Table 3 Comparison of Central Corneal Thickness Pre-Operatively and Post-Operatively in Modified Prechop and Phaco-Chop Groups

\begin{tabular}{|l|c|c|c|}
\hline & Prechop Group & Phaco-chop Group & P \\
\hline Pre-operative $(\mu \mathrm{m})$ & $517 \pm 28$ & $517 \pm 29$ & 0.990 \\
\hline I-month post-operative & & & \\
CCT $(\mu \mathrm{m})$ & $522 \pm 30$ & $524 \pm 35$ & 0.662 \\
Change $(\%)$ & $0.9 \pm 2.3 \%$ & $1.3 \pm 4.0 \%$ & 0.404 \\
\hline 3-month post-operative & & & \\
CCT $(\mu \mathrm{m})$ & $519 \pm 28$ & $517 \pm 30$ & 0.618 \\
Change $(\%)$ & $0.4 \pm 1.9 \%$ & $0.0 \pm 2.9 \%$ & 0.272 \\
\hline
\end{tabular}

and after 6 months or in posterior capsule rupture rates and endothelial cell loss after 6 months between conventional cataract surgery and FLACS. ${ }^{18}$ Moreover, FLACS has resulted in more frequent anterior capsular ruptures. The costeffectiveness of the FLACS is still controversial.

The modified prechopper technique aims to eliminate the use of a phacoemulsification tip during the fragmentation stage. The two classic prechoppers, Akahoshi and Inamura prechoppers, can cut through LOCS III N1 to LOCS III N3 dense lenses without significant zonular stress, and completely separate two stages of the procedure. However, in difficult cases, such as mature or hypermature hard endonucleus with LOCS III N4 and above cataracts, and weak zonule, a prechopping technique using classic instruments can increase the risk of the posterior capsule injury and zonular lysis.

The modified $1.7 \mathrm{~mm}$ neck, prechopper employs the combination of the mechanical advantages of an axe and the beak of an eagle.

The axe is an example of a simple machine that acts as a wedge and changes the direction of the input force. It functions by converting a force applied to its blunt end into forces perpendicular to its inclined surfaces. A wedge applies more force to the object (output force) than the user applies to the wedge (input force) (Figure 7). The handle of the axe also acts as a lever allowing the user to increase the force at the cutting edge. The ideal mechanical advantage (IMA) is the ratio of the depth of penetration to the width of the wedge, and can be calculated by the length of the blade divided by its width. The IMA value of the HI Kim-Inamura prechopper is $0.16 \mathrm{~mm}$ divided by $1.7 \mathrm{~mm}$, which is 10.625 .

It is very difficult to break wood across the grain because it involves fracturing the tracheids. In contrast, splitting wood along the grain is relatively easy, especially radially down the center of the branch, as this involves separating the tracheid cells. Likewise, a human crystalline lens is a mass of tightly packed lens fibers. The structure of the lens is like the structure of an onion (Figure 8A and B) ${ }^{19}$ Therefore, splitting along the lens orientation allows fracturing the nucleus much more easily. The beak of eagle tapers down to a sharp, hooked point. It helps digging and ripping the flesh of prey into small pieces. The combination of the mechanical properties of an axe and the principle of a beak can multiply chopping force while maintaining a stable fixation. 

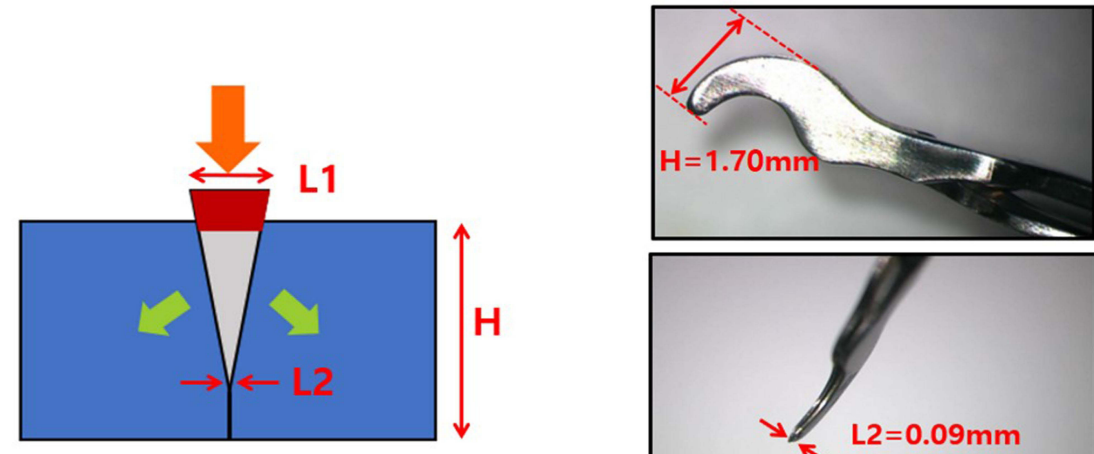

What is the IMA of the wedge shown?

IMA $=1.70 \mathrm{~mm} /(0.25-0.09)=1.70 / 0.16=10.625$
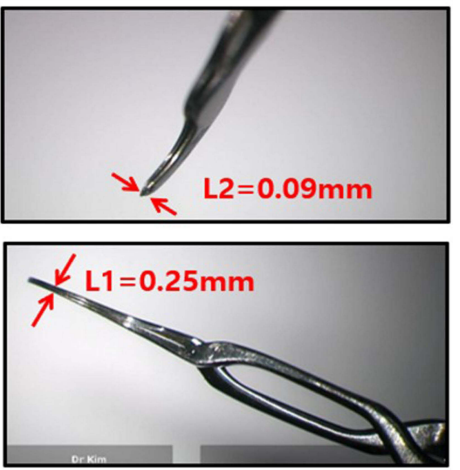

Figure 7 The illustration of wood splitting and wedge.

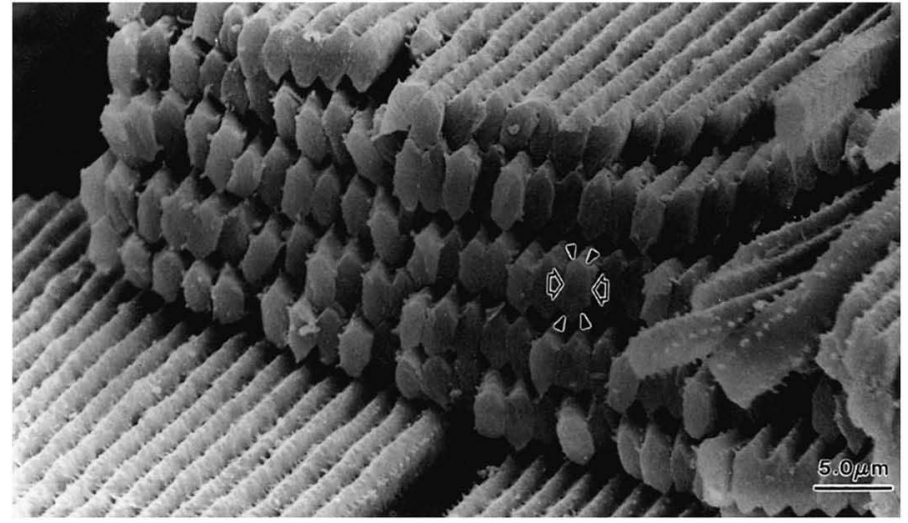

A

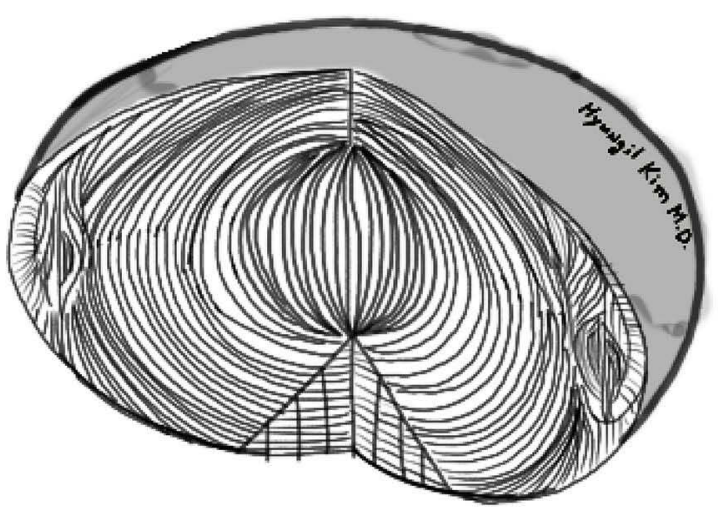

B

Figure 8 (A) Scanning electron micrograph showing the orderly arrangement of hexagonal lens fibers (arrows) in the vertebrate lens. Reprinted from Journal of NeuroOphthalmology, 24(4). Kaufman PL, Alm A. Adler's Physiology of the Eye: Clinical Application. I0th ed. St. Louis, Mosby: Journal of Neuto-Ophthalmology; 348, Copyright 2004, with permission from Elsevier. ${ }^{19}$ (B) The schematic of the human crystalline lens.

Until the 3-month post-operative follow-up, there were no significant differences in the absolute ECCs, losses of ECC, absolute CCT, and change in CCT between the modified prechop group and the modified the phaco-chop group. This shows that the prechop technique, which is relatively easy for beginners, is a versatile and effective maneuver.

In summary, the one-hand prechop using only a modified prechopper is suitable for soft nuclei from LOCS III N1 to N3 cataract. The counter prechop is suitable for hard nuclei from LOCS III N4 to N6 cataract.

The prechop technique using a modified eagle type prechopper is feasible and can be performed in a wide range of cataracts, including soft lenses (Video 1), LOCS III N1 to N4 cataracts (ideo 2), and weak zonule cataracts (Video 4). Modified counter prechopping with a universal chopper can be performed over LOCS III N5 (Video 5), white cataracts (Video 6A and B), and even mature or hypermature cataracts with nuclear opalescence LOCS III N6 (Video 7). In difficult cases, such as various pupil sizes including small pupil, the modified prechop technique also can be performed using iris retractors (Video 8).

Although the prechop technique using a modified eagle type prechopper is a versatile and effective maneuver, it is not suitable for some cases such as zonular lysis, extremely dense cataracts, incomplete continuous curvilinear capsulorhexis 
(CCC), and hypermature cataracts with little to no cortical layer due to emulsification. In the exceptional cases mentioned above, conventional cataract surgery including sculpting and chopping with US energy may be required to facilitate lens disassembly.

A limitation of our study is that no comparison has been made in the mean cumulative dissipated energy. The settings we use are linear pulse mode, which does not provide the mean ultrasound power required to calculate the cumulative dissipated energy. In the future, a follow-up study that can compare total ultrasound energy is required.

In conclusion, the counter prechop technique using the modified prechopper and universal chopper is an alternative surgical procedure that can be performed safely in LOCS III N1 to N6 nuclei, overripe nuclei, small pupil cases, and fragile suspensory ligaments.

\section{Disclosure}

No author has a financial or proprietary interest in any material or method mentioned in this study.

\section{References}

1. Kelman CD. Phaco-emulsification and aspiration. A new technique of cataract removal. A preliminary report. Am J Ophthalmol. 1967;64(1):23-35. doi:10.1016/0002-9394(67)93340-5

2. Akahoshi T. Phaco prechop: manual nucleofracture prior to phacoemulsification. Oper Tech Cataract Refract Surg. 1998;1:69-91.

3. Gimbel HV. Divide and conquer nucleofractis phacoemulsification: development and variations. J Cataract Refract Surg. 1991;17(3):281-291. doi:10.1016/s0886-3350(13)80824-3

4. Koch PS, Katzen LE. Stop and chop phacoemulsification. J Cataract Refract Surg. 1994;20(5):566-570. doi:10.1016/s0886-3350(13)80239-8

5. Fine IH. The chip and flip phacoemulsification technique. J Cataract Refract Surg. 1991;17(3):366-371. doi:10.1016/s0886-3350(13)80835-8

6. Berger A, Contin IN, Nicoletti G, et al. Middle prechop: fracturing the middle portion of the nucleus. J Cataract Refract Surg. 2012;38(4):564-567. doi:10.1016/j.jcrs.2012.02.001

7. Chen X, Liu B, Xiao Y, et al. Cystotome-assisted prechop technique. J Cataract Refract Surg. 2015;41(1):9-13. doi:10.1016/j.jcrs.2014.11.015

8. Zhao Y, Li J, Yang K, Zhu S. A prechop technique using a reverse chopper. J Invest Surg. 2019;32(3):199-207. doi:10.1080/ 08941939.2017.1391358

9. Kim DB. Double-chop: modified-chop technique eliminating ultrasonic energy and vacuum for lens fragmentation. $J$ Cataract Refract Surg. 2016;42(10):1402-1407. doi:10.1016/j.jcrs.2016.08.014

10. Nagy Z, Takacs A, Filkorn T, Sarayba M. Initial clinical evaluation of an intraocular femtosecond laser in cataract surgery. J Refract Surg. 2009;25 (12):1053-1060. doi:10.3928/1081597X-20091117-04

11. Tak'acs AI, Kov'acs I, Mih'altz K, et al. Central corneal volume and endothelial cell count following femtosecond laser-assisted refractive cataract surgery compared to conventional phacoemulsification. J Refract Surg. 2012;28(6):387-391. doi:10.3928/1081597X-20120508-02

12. Mastropasqua L, Toto L, Mattei PA, et al. Optical coherence tomography and 3-dimensional confocal structured imaging system-guided femtosecond laser capsulotomy versus manual continuous curvilinear capsulorhexis. J Cataract Refract Surg. 2014;40(12):2035-2043. doi:10.1016/j.jcrs.2014.05.032

13. Reddy KP, Kandulla J, Auffarth GU. Effectiveness and safety of femtosecond laser-assisted lens fragmentation and anterior capsulotomy versus the manual technique in cataract surgery. $J$ Cataract Refract Surg. 2013;39(9):1297-1306. doi:10.1016/j.jcrs.2013.05.035

14. Conrad-Hengerer I, Al Juburi M, Schultz T, et al. Corneal endothelial cell loss and corneal thickness in conventional compared with femtosecond laser-assisted cataract surgery: three-month follow-up. J Cataract Refract Surg. 2013;39(9):1307-1313. doi:10.1016/j.jcrs.2013.05.033

15. Mayer WJ, Klaproth OK, Hengerer FH, Kohnen T. Impact of crystalline lens opacification on effective phacoemulsification time in femtosecond laser-assisted cataract surgery. Am J Ophthalmol. 2014;157(2):426-432. doi:10.1016/j.ajo.2013.09.017

16. Abell RG, Vote BJ. Cost-effectiveness of femtosecond laser-assisted cataract surgery versus phacoemulsification cataract surgery. Ophthalmology. 2014;121(1):10-16. doi:10.1016/j.ophtha.2013.07.056

17. Dick HB, Gerste RD. Plea for femtosecond laser pre-treatment and cataract surgery in the same room. J Cataract Refract Surg. 2014;40 (3):499-500. doi:10.1016/j.jcrs.2014.01.005

18. Kolb CM, Shajari M, Mathys L, et al. Comparison of femtosecond laser-assisted cataract surgery and conventional cataract surgery: a metaanalysis and systematic review. J Cataract Refract Surg. 2020;46(8):1075-1085. doi:10.1097/j.jcrs.0000000000000228

19. Kaufman PL, Alm A. Adler's Physiology of the Eye: Clinical Application. 10th ed. St. Louis, Mosby: Journal of Neuro-Ophthalmology; 2004;24 (4):348.

Clinical Ophthalmology

Dovepress

\section{Publish your work in this journal}

Clinical Ophthalmology is an international, peer-reviewed journal covering all subspecialties within ophthalmology. Key topics include: Optometry; Visual science; Pharmacology and drug therapy in eye diseases; Basic Sciences; Primary and Secondary eye care; Patient Safety and Quality of Care Improvements. This journal is indexed on PubMed Central and CAS, and is the official journal of The Society of Clinical Ophthalmology (SCO). The manuscript management system is completely online and includes a very quick and fair peer-review system, which is all easy to use. Visit http://www. dovepress.com/testimonials.php to read real quotes from published authors.

Submit your manuscript here: https://www.dovepress.com/clinical-ophthalmology-journal 